\title{
THE SECOND VARIATION FORMULA FOR HARMONIC MAPPINGS
}

\author{
R. T. SMITH 1
}

ABSTRACT. The formula of the title is computed, and is used to calculate the index and nullity in several cases.

Introduction. The variational characterization of a harmonic mapping of Riemannian manifolds $f: N \rightarrow M$ states that $f$ is harmonic iff the Dirichlet energy $E(f)$ of the map is stable to first order with respect to variations of $f$ [ES]. Here $E(f)=1 / 2 \int_{N}|d f|^{2} * 1$, where $N$ is taken to be closed and oriented. The object of this paper is to give a formula for the second variation of the energy and use it to calculate the index and nullity of some harmonic maps. For the (nontrivial!) case of the identity map, there are close relations with certain infinitesimal transformation groups. As a by-product we are led to a unified interpretation (in $\$ 2$ ) of a differential operator which has turned up in varied geometrical contexts before (e.g. [BY], [L1], [N1], [YN]). Examples are also given to show that the Morse theory of a harmonic map can be quite pathological. Finally, we note that the second variation formula for minimal submanifolds (i.e., the volume functional) can be found in [HE].

The results of this paper appear in the author's thesis [SMI], and I would like to thank David Elworthy and James Eells for their helpful suggestions. Since this article was accepted for publication, the second variation formula has appeared elsewhere (E. Mazet, La formule de la variation seconde de l'énergie au voisinage d'une application harmonique, J. Differential Geometry 8 (1973), 279-296), in which some different applications are developed.

1. The second variation formula. Let $f: N \rightarrow M$ be a smooth map, and $f_{s, t}$ a smooth 2-parameter variation of $f$ with $\partial f / \partial s=v$ and $\partial f / \partial t=w$ at $s=t=0$.

Received by the editors May 23, 1973 and, in revised form, October 9, 1973. AMS (MOS) subject classifications (1970). Primary 58E 15, 53C20.

Key words and phrases. Harmonic mapping, energy functional, Morse theory, infinitesimal transformation group.

${ }^{1}$ Research supported by Marshall Scholarship and NSF Graduate Fellowship. 
Proposition 1.1. If $f: N \rightarrow M$ is harmonic, the Hessian of the energy functional at $f$ is given by

$$
H(v, w)=\int_{N}\left\langle\nabla_{f} v, \nabla_{f} w\right\rangle-\left\langle\rho_{f}(v), w\right\rangle
$$

Remark. $\nabla_{f}$ is the induced connection on $f * T M$, and $\rho_{f}(v)=$ Trace $R_{M}(d f, v) d f$, where $R_{M}$ is the curvature tensor on $M$. Our reference for induced connections, etc., is Eliasson [El], whose sign convention for $R$ is opposite to ours. These calculations depend heavily on his work.

Proof of 1.1. Choose a variation $f_{s, t}$ as above. Then $f_{s, t}$ defines a map $F: \mathbf{R}^{2} \times N \rightarrow M$, and

$$
E\left(f_{s, t}\right)=\frac{1}{2} \int_{N}\langle d F(-), d F(-)\rangle
$$

where (-) will denote arguments in TN. Then as all Riemannian manifolds are assumed to carry their Levi-Civita connections, we have

$$
\begin{gathered}
\frac{\partial E}{\partial t}=\int_{N}\left\langle\nabla d F\left(\frac{\partial}{\partial t},-\right), d F(-)\right\rangle=\int_{N}\left\langle\nabla d F\left(-, \frac{\partial}{\partial t}\right), d F(-)\right\rangle, \\
\frac{\partial^{2} E}{\partial s \partial t}=\int_{N}\left\langle\nabla^{2} d F\left(\frac{\partial}{\partial s},-, \frac{\partial}{\partial t}\right), d F(-)\right\rangle+\left\langle\nabla d F\left(-, \frac{\partial}{\partial t}\right), \nabla d F\left(-, \frac{\partial}{\partial s}\right)\right\rangle .
\end{gathered}
$$

Note that $\nabla^{2} d F(\partial / \partial s,-, \partial / \partial t)=\nabla^{2} d F(-, \partial / \partial s, \partial / \partial t)-\left(R_{L}(\partial / \partial s,-) d F\right) \partial / \partial t$ where $R_{L}$ is the curvature for the bundle $L\left(T\left(\mathbf{R}^{2} \times N\right), F * T M\right)$. From [El] we get

$$
\begin{aligned}
\left(R_{L}\left(\frac{\partial}{\partial s},-\right) d F\right) \frac{\partial}{\partial t} & =R_{F * T M}\left(\frac{\partial}{\partial s},-\right) \frac{\partial F}{\partial t}-d F\left(R_{\mathbf{R}^{2} \times N}\left(\frac{\partial}{\partial s},-\right) \frac{\partial}{\partial t}\right) \\
& =R_{M}\left(\frac{\partial F}{\partial s}, d F(-)\right) \frac{\partial F}{\partial t} .
\end{aligned}
$$

Note also that $\nabla d F(\partial / \partial s, \partial / \partial t)$ is a field along $F$, so that at $s=t=0$ we have

$$
\int_{N}\left\langle\nabla^{2} d F\left(-, \frac{\partial}{\partial s}, \frac{\partial}{\partial t}\right), d F(-)\right\rangle=0
$$

as $f_{0,0}$ is harmonic. Thus we conclude

$$
\left.\frac{\partial^{2} E}{\partial s \partial t}\right|_{s=t=0}=\int_{N}\left\langle\nabla_{f} w, \nabla_{f} v\right\rangle-\left\langle R_{M}(v, d f) w, d f\right\rangle .
$$


In general, however, the form $\langle R(X, Y) Z, W\rangle$ is antisymmetric in $(X, Y)$ and in $(Z, W)$. (See Milnor [M, p. 53].): This proves the proposition.

An immediate corollary is that if all sectional curvatures of $M$ are nonpositive, then $H(v, v) \geq 0$ for all variations $v$; this indicates that every such harmonic map is a local minimum of the energy. In fact, a much stronger statement is true: if $f: N \rightarrow M$ is a harmonic map and $M$ (complete) has nonpositive sectional curvature, then $f$ is an absolute minimum of the energy in its homotopy class. The proof is simply that any smooth map $f_{0}$ in this class is smoothly homotopic to a harmonic map $f_{\infty}$ via the heat equation (using Eells-Sampson [ES] and Hartman [H, p. 674]). As energy decreases along a trajectory of the heat equation [ES, p. 135] $E\left(f_{\infty}\right) \leq E\left(f_{0}\right)$. Furthermore, Hartman has shown that $f_{\infty}$ must be homotopic to $f$ through harmonic maps, and consequently that $E(f)=E\left(f_{\infty}\right)[\mathrm{H}, \mathrm{p} .675]$. Hence $E(f)$ is an absolute minimum.

An absolute minimum property is also satisfied by holomorphic mappings of Kaehler manifolds. (Such maps are necessarily harmonic; see [ES, p. 118].) For this we need the following theorem of Lichnerowicz: if $f: N \rightarrow M$ is a map of Kaehler manifolds ( $N$ compact), we can write $d f=d f_{(0,1)}+d f_{(1,0)}$, the decomposition into complex linear and conjugate linear parts. Accordingly there is a decomposition of the energy $E(f)=E^{\prime}(f)+E^{\prime \prime}(f)$. (Hence $f$ is holomorphic iff $\left.E^{\prime \prime}(f)=0.\right)$ Let $K(f)$ be the difference $E^{\prime}(f)-E^{\prime \prime}(f)$

Proposition 1.3 [L2, p. 376]. If $f_{t}$ is an arbitrary smooth homotopy of maps between Kaehler manifolds $N$ and $M$, with $N$ closed, then $K\left(f_{t}\right)$ is constant.

Corollary 1.4. A holomorphic (or antiholomorphic) map of Kaehler manifolds with closed domain gives an absolute minimum of the energy in its homotopy class.

By applying the divergence theorem, as in [E2, p. 64]. we see that the Hessian can also be written as

$$
H(v, w)=\int_{N}\left\langle-\Delta_{f} v-\rho_{f} v, w\right\rangle=\int_{N}\left\langle J_{f} v, w\right\rangle
$$

where $\Delta_{f} v=$ Trace $\nabla_{f}^{2} v$. Hence by symmetry and ellipticity

$$
\begin{aligned}
\operatorname{Null}(f) & =\operatorname{dim} \operatorname{ker} J_{l f}<\infty, \\
\operatorname{index}(f) & =\#\left\{\text { eigenvalues }\left(J_{f}\right)<0\right\}<\infty .
\end{aligned}
$$

In analogy with the theory of geodesics, we call $v$ a Jacobi field if $v \in \operatorname{ker} J_{f} \cdot$ Note that if $f_{t}$ is a variation of $f$ through harmonic maps, then 
$v=\partial f /\left.\partial t\right|_{t=0}$ is trivially a Jacobi field.

The converse is not necessarily true, even for closed geodesics; further, it is probably not even true locally for a general harmonic map (see Example 3.4). However, it is true that if a harmonic map $f$ is restricted to a small disc in its domain, then $f$ gives a local minimum of the energy among mappings with the same boundary values. That is, general elliptic theory says that the eigenvalues of $J_{f}$ on fields $v$ which vanish on the boundary will all be positive (cf. [SMA, Lemma 7]).

Regarding the nullity of a harmonic map, let us define the killing nullity as

$$
\operatorname{Null}_{k}(f)=\operatorname{dim} \operatorname{span}(\mathbf{i}(M)), d f(\mathbf{i}(N)) .
$$

Here elements of $\mathbf{i}(M)$ (infinitesimal isometries) and $d f(\mathbf{i}(N)$ ) are considered as variation fields along $f$. They are clearly Jacobi fields, as they arise from the composition of $f$ with 1 parameter groups of isometries. It will be convenient to discount these trivial harmonic variations and define a reduced nullity

$$
\operatorname{Null}_{r}(f)=\operatorname{Null}(f)-\operatorname{Null}_{k}(f) .
$$

The qualitative problems of interest may now be stated: (1) what is index $(f)$ ? (2) If null $r(f) \neq 0$, do all Jacobi fields arise from a variation of $f$ through harmonic maps? Let us say that $f$ is a generate harmonic map if this is the case. It should be noted that nondegeneracy, in the sense of Palais-Smale $[\mathrm{P}]$, is a stronger condition which can never be satisfied on the usual Sobolev manifolds if the domain has dimension $\geq 2$ (cf. [SMI, Appendix]).

2. Relations with transformation groups and the spectrum。 It was first observed in [ES] that the identity on $S^{n}$ is not a local minimum of the energy for $n \geq 3$. Variations of the identity on a general closed oriented Riemannian manifold $M$ are simply vector fields, and the second variation operator becomes

$$
J v=-\Delta v-\rho(v)
$$

Here $\Delta v=$ Trace $\nabla^{2} v$ and $\rho$ is the Ricci tensor, considered as a linear map. The Hodge Laplacian $\Delta_{H}$ (acting on vector fields via duality) satisfies the well-known formula (cf. [BY] or [YN, p.56]).

$$
\Delta_{H} v=-\Delta v+\rho(v) \text {. }
$$

Jacobi fields, or solutions of $J v=0$, are studied in [YN] and there labelled 
"geodesic vector fields." (The terminology was suggested by the fact that the equation of a totally geodesic vector field is $\nabla^{2} v+R(-, v)-=0$.). Y ano and Nagano conjectured that the flow of such a field would preserve geodesics "on average." More precisely, does the flow of a Jacobi field consist of harmonic mappings? This question in unanswered for closed manifolds; furthermore, the next two examples indicate that (in contrast to the case of totally geodesic fields and flows) this problem is not local.

Example 2.2. Define a vector field on $\mathbf{R}^{n}$, for $n \geq 2$, by $v(x)=$ $\left(\cos x_{1} \cosh x_{2}, 0, \cdots, 0\right)$. Then $v$ is a Jacobi field, but one can check that the flow of $v$ does not consist of harmonic maps.

Example 2.3. Suppose $M$ is flat and closed. Then a Jacobi field satisfies $0=\int_{M}\langle\Delta v, v\rangle=-\int_{M}|\nabla v|^{2}$ so that $\nabla v=0$. Hence $v$ is a parallel vector field and thus an infinitesimal isometry.

Another case in which integration can be used to show that the identity is a generate critical point is

Example 2.4 ([L1], [YN, p. 57]). Let $M$ be a closed Kaehler manifold and $v$ a vector field with $J v=0$. Then $v$ is an infinitesimal analytic transformation.

Relations between the operator $J$ and the Lie algebra of infinitesimally conformal fields (c) are given in [L1]. A formula useful in this regard can be found in [BY, p. 57].

$$
\int_{M}\langle J v, v\rangle=\int_{M} \frac{1}{2}|L v g|^{2}-\delta(v)^{2}
$$

Here $L_{v} g$ is the Lie derivative of the metric w.r.t. $v$ and $\delta$ is the divergence. Thus

Proposition 2.6. If $M$ is closed and oriented of dimension $\geq 3$, index $\left(\operatorname{id}_{M}\right) \geq \operatorname{dim}(\mathbf{c} / \mathrm{i})$.

Proof. A field in c satisfies $L_{v} g=(2 / n) \delta(v) g$, hence $\left|L_{v} g\right|^{2}=$ $(4 / n) \delta(v)^{2}$. The conclusion is immediate from (2.5).

Corollary 2.7. Any compact homogeneous space of dimension $\geq 3$ can be given a metric for which $\mathrm{id}_{M}$ is not a local minimum of the energy.

Proof. If $f_{t}$ is a one-parameter group of isometries for the metric $g$, then there is a new metric under which the $f_{t}$ are only conformal (see [KN, p. 310])。

Now suppose $M$ is Einstein, with $\rho=c g$. Then

$$
J=\Delta_{H}-2 c I
$$


and we can relate the index and nullity of $\mathrm{id}_{M}$ to the spectrum of $M$ (the eigenvalues of $\Delta_{H}$ on functions). If $\lambda(r)=\#\{$ eigenvalues $\lambda: 0<\lambda<r\}$ and $m(r)$ is the multiplicity of $r$ (with $m(0)$ defined to be 0 ), we have

Proposition 2.11. Let $M$ be a closed oriented Einstein manifold with $\rho=c g$ for some scalar $c$. Then

(a) $\operatorname{index}\left(\mathrm{id}_{M}\right)=\lambda(2 c)$,

(b) $\operatorname{null}\left(\mathrm{id}_{M}\right)=\operatorname{dim}(\mathrm{i})+m(2 c)$,

(c) null $_{r}\left(\right.$ id $\left._{M}\right)=m(2 c)$.

The proof is basically a standard argument using the Hodge decomposition of a vector field as a sum of a gradient field and a divergence-free field, and also Yano's formula (2.5). For example, see [YN, Theorem 5.3] for (b).

Example 2.12. $S^{n}$ with the Euclidean metric is an Einstein space with $\rho=(n-1) g$. The spectrum is $\left\{\lambda_{k}=k(k+n-1): k \geq 0\right\}$. Thus $n$ is the only nonzero eigenvalue which is $\leq 2(n-1)$.

The corresponding eigenfunctions are the restrictions of linear forms on $\mathbf{R}^{n+1}$ (harmonic polynomials of degree one), so the multiplicity is evidently $n+1$. Hence

$$
\begin{aligned}
\operatorname{index}\left(\text { id }_{S^{n}}\right) & =n+1, & & n \geq 3, \\
& =0, & & n=1,2, \\
\text { Null } \left._{r} \text { id }_{S^{n}}\right) & =0, & & n \neq 2, \\
& =3, & & n=2 .
\end{aligned}
$$

We remark that the gradient of a linear form on $S^{n}$ is an element of c and is perpendicular to i. Hence the connection between Proposition 2.6 and Proposition 2.11 is exact for spheres. (It was actually the flow of one of these fields which was observed to be energy decreasing in [ES].). Finally, as conformal maps are harmonic in 2 dimensions, we conclude that id $s^{n}$ is a generate critical point for all $n$.

With regard to certain other Einstein spaces, we can make use of a table of spectra given by Nagano [N1] to conclude

Proposition 2.13. index $\left(\mathrm{id}_{M}\right)=0$ for the classical compact irreducible symmetric spaces, with the following exceptions:

(i) $S^{n}, n \geq 3$; (ii) $\mathrm{Sp}(p+q) / \mathrm{Sp}(p) \times \mathrm{Sp}(q)$; (iii) $\mathrm{SU}(2 m) / \mathrm{Sp}(m), m>1$.

Note further that $\mathbf{c}=\mathbf{i}$ for all such spaces except spheres [N2].

Hence the estimate in Proposition 2.6 cannot be replaced by equality. 
3. Pathologies. Suppose both : $N$ and $M$ are closed, and $\pi: N \times M \rightarrow M$ is the projection. If $J$ is the Jacobi operator on $M$, with eigenvectors $J v_{j}=$ $\lambda_{j} v_{j}$, and if $f_{i}$ are eigenfunctions of the Laplacian of $N, \Delta_{N} f_{i}=\mu_{i} f_{i}$, then a short computation shows

Proposition 3.1. The variations of $\pi$ defined by $a_{i j}(n, m)=f_{i}(n) v_{j}(m)$ satisfy $J_{\pi}\left(a_{i j}\right)=\left(\mu_{i}+\lambda_{j}\right) a_{i j}$.

Corollary 3.2.

$$
\text { index }(\pi) \geq \sum_{\lambda_{j}<0} m_{M}\left(\lambda_{j}\right) \lambda_{N}\left(\left|\lambda_{j}\right|\right), \quad \operatorname{null}_{r}(\pi) \geq \sum_{\lambda_{j}<0} m_{M}\left(\lambda_{j}\right) m_{N}\left(\left|\lambda_{j}\right|\right) .
$$

Here $m_{M}\left(\lambda_{j}\right)$ is the multiplicity of $\lambda_{j}$ as an eigenvalue of the Jacobi operator on $M$, and $\lambda_{N}\left(\left|\lambda_{j}\right|\right)$ is the number of eigenvalues of $\Delta_{N}$ which are less than $\left|\lambda_{j}\right|$, this time including 0 .

Corollary 3.3. Let $M$ be a closed Riemannian manifold for which index $\left(\mathrm{id}_{M}\right)>0$, and let $N$ be any closed Riemannian manifold of dimension $\geq 1$. If $\pi: N \times M \rightarrow M$ is the trivial bundle:

(a) by making a scalar change of metric on $N$, we can make the Morse index of $\pi$ arbitrarily large.

(b) if further the multiplicities in the spectrum of $N$ satisfy $\lim _{n \rightarrow \infty} m\left(\lambda_{n}\right)=\infty$, we can similarly make the reduced nullity of $\pi$ arbitrarily large.

Proof. Multiplying the metric of $N$ by $c$ multiplies the spectrum of $N$ by $1 / c$. For suitable large $c$ we get (a) and (b).

Example 3.4. Let $M=S^{n}$, for $n \geq 3$, and let $N=S^{p}$. If $v$ is a conformal gradient field on $S^{n}$ and $f$ is a harmonic polynomial of homogeneity $k$ on $S^{p}$, then by a scalar change of metric on $S^{p}$ we can assume that $w(x, y)=$ $f(x) v(y)$ is a Jacobi field for $\pi$. It seems quite unlikely that these fields arise from a variation of $\pi$ through harmonic maps. Since the harmonic polynomials satisfy the condition in (b) of the above corollary, for $p \geq 2$, it appears that $\pi$ can be given an arbitrarily large degeneracy as well as a massive index. Hence the innocuous map $\pi$ is completely pathological from the standpoint of Morse theory.

\section{REFERENCES}

[BY] K. Yano and S. Bochner, Curvature and Betti numbers, Ann. of Math. Studies, no. 32, Princeton Univ. Press, Princeton, N. J., 1953. MR 15, 989.

[ES] J. Eells and J. H. Sampson, Harmonic mappings of Riemannian manifolds, Amer. J. Math. 86 (1964), 109-160. MR 29 \#1603. 
[E1] H. Eliasson, On the geometry of manifolds of maps, J. Differential Geometry 1 (1967), 169-194. MR 37 \#2268.

[E2] - Variational integrals in fibre bundles, Proc. Sympos. Pure Math., vol. 16, Amer. Math. Soc., Providence, R. I., 1970, pp. 67-89. MR 42 \#2507.

[H] .P. Hartman, On homotopic harmonic maps, Canad. J. Math. 19 (1967), 673687. MR $35 \# 4856$.

[HE] R. Hermann, The second variation for variational problems in canonical form, Bull. Amer. Math. Soc. 71 (1965), 145-148. MR 30 \#2436.

[KN] S. Kobayashi and K. Nomizu, Foundations of differential geometry. Vol. II, Interscience Tracts in Pure and Appl. Math., no. 15, Vol. II, Interscience, New York, 1969. MR 38 \#6501.

[L1] A. Lichnérowicz, Géométrie des groupes de transformations, Travaux et Recherches Mathématiques, III, Dunod, Paris, 1958. MR 23 \# A1329.

[L2] Applications harmoniques et variêtés kähleriennes, Symposia Mathematica, Vol. III (INDAM, Rome, 1968/69), Academic Press, London, 1970, pp. 341-402. MR 41 \#7598.

[M] J. Milnor, Morse theory, Ann. of Math. Studies, no. 51, Princeton Univ. Press, Princeton, N. J., 1963. MR 29 \#634.

[N1] T. Nagano, On the minimum eigenvalues of the Laplacians in Riemannian manifolds, Sci. Papers Coll. Gen. Ed. Univ. Tokyo 11 (1961), 177-182. MR 26 \#1830.

[N2] On conformal transformations of Riemannian spaces, J. Math.

Soc. Japan 10 (1958), 76-93. MR 22 \# 1859.

[P] R. S. Palais, Morse theory on Hilbert manifolds, Topology 2 (1963), 299-340. MR 28 \# 1633.

[SMA] S. Smale, On the Morse index theorem, J. Math. Mech. 14 (1965), 1049-1055. MR $31 \# 6251$.

[SMI] R. T. Smith, Thesis, Warwick University, 1972.

[YN] K. Yano and T. Nagano, On geodesic vector fields in a compact orientable Riemannian space, Comment. Math. Helv. 35 (1961), 55-64. MR 23 \#A2164.

DEPARTMENT OF MATHEMATICS, MASSACHUSETTS INSTITUTE OF TECHNOLOGY. CAMBRIDGE, MASSACHUSETTS 02139

Current address: Department of Mathematics, Columbia University, New York, New York 10027 